\title{
Neuordnung der Verantwortungen in der Heilkunde aus Sicht der Ärzteschaft
}

\author{
Theodor Windhorst
}

(C) Springer-Verlag Wien 2012

Chronisch und multimorbid kranke alte Menschen haben einen hochkomplexen medizinischen, pflegerischen und psychosozialen Versorgungsbedarf, der eine optimale Zusammenarbeit der verschiedenen Professionen erfordert. Ärzte und Pfleger müssen sich als Team verstehen und alle Professionen partnerschaftlich zusammenarbeiten.

Maßstab für eine Fortentwicklung der Aufgabenverteilung zwischen den Gesundheitsberufen muß aber in jedem Fall das Primat der Qualität und Sicherheit der Patientenversorgung sein. Die Sicherheit der Patienten geht allen anderen Gesichtspunkten vor. Sie darf weder etwaigen personellen Engpässen geopfert, noch darf sie zum Spielball von isolierten Sichtweisen der beteiligten Berufsgruppen werden. Auch unter den derzeit begrenzten finanziellen Mitteln muss der Patient im Zentrum einer gerechten Versorgung stehen.

Neben einer Arztentlastung in der zunehmend bürokratisch reglementierten Versorgungswelt sollte auch eine Neudefinition der Pflegefachberufe in den Fokus rücken.
Allerdings wird die Einführung einer neuen, nichtärztlichen Versorgungsebene die Probleme in unserem Gesundheitswesen nicht lösen können. Die Substitution ärztlicher Arbeit durch andere Berufe ist weder kostengünstiger noch qualitativ besser. Ziel muss eine gerechte und faire Entgelt- und Arbeitszeitsituation für alle Berufsgruppen sein.

Die Ärzteschaft befürwortet eine Delegation in der medizinischen Versorgung an Gesundheitsfachberufe unter der Voraussetzung der therapeutischen Gesamtverantwortung des Arztes, bei dem auch die Budgetverantwortung liegen muss. Einflussfaktoren auf die Delegationsfähigkeit sind Komplexität der Aufgabe, Gefahrenpotential und die Qualifikation des Delegationsempfängers.

Die Bewältigung zukünftiger Aufgaben in der Patientenversorgung vor dem Hintergrund der demographischen Entwicklung und des medizinischen Fortschrittes kann nur in einer verantwortungsvollen Kooperation der verschiedenen Berufsgruppen gelingen.

T. Windhorst $(\bowtie)$

Präsident, Ärztekammer Westfalen-Lippe, Münster 